\title{
MEASUREMENT OF HIGH-ENERGY REFLECTED NEUTRON FROM ALUMINIUM WITH THRESHOLD DETECTORS
}

\author{
Lu Xinxin * \\ Institute of Nuclear Physics and Chemistry, CAEP \\ P.O.Box 919-213 Mianyang of Sichuan Prov, 621900, China \\ E-mail: luxinxin5187@sohu.com \\ Liu Rong \\ Institute of Nuclear Physics and Chemistry, CAEP \\ P.O.Box 919-213 Mianyang of Sichuan Prov, 621900, China \\ Jiang Li \\ Institute of Nuclear Physics and Chemistry, CAEP \\ P.O.Box 919-213 Mianyang of Sichuan Prov, 621900, China \\ Wang Mei \\ Institute of Nuclear Physics and Chemistry, CAEP \\ P.O.Box 919-213 Mianyang of Sichuan Prov, 621900, China
}

\begin{abstract}
Absolute activation reaction rates of high-energy neutrons from an $\mathrm{Al}$ reflector are obtained using six different high-threshold neutron-activation detectors. For the different activation detectors the shape of the rates as function of the measurement angle is rather similar. Measured reflectivities are also obtained as the ratio of measured data and range from 1.0 to 1.14. The intensity of the reflected high-energy neutron field is high at large angles and smaller towards smaller angles. With increasing thresholds of the activation detectors, the reflectivities decrease. Evaluated total uncertainties of the measured reflectivities are 3.4\% 4.2\%. Calculated results of reflectivities agree with the experimental results at the majority of the measuring points.
\end{abstract}

International Workshop on Fast Neutron Detectors

University of Cape Town, South Africa

April 3-6, 2006

\footnotetext{
${ }^{*}$ Speaker
} 


\section{Introduction}

Reflected neutrons may cause critical safety conditions in nuclear material stockpiles when they act on nuclear materials and they also affect the design of reactor shieldings. Some laboratories have studied reflected neutrons from different materials such as concrete, Iron, Carbon, etc $[1,2,3]$. Most of the available information is, however, based on calculations and only few experimental data are available. In most of these experiments the reflected neutron yield is studied for concrete slabs, investigations of other materials or other structures are scarce. Aluminium is a common construction material in nuclear facilities. Therefore, measuring reflected neutrons from $\mathrm{Al}$ is very important. For studying the high-energy reflected neutrons from $\mathrm{Al}$, six different threshold detectors made of $\mathrm{Fe}, \mathrm{Al}, \mathrm{Nb}, \mathrm{F}, \mathrm{Zr}$, and $\mathrm{Cu}$ are used to measure high-threshold activation reaction rates. From these activation data reflectivities of materials bombarded by D-T neutrons are derived.

\section{Measurement method}

The neutron-activation method is used to measure reflected high-energy neutrons from Al. For a single nuclide the activation reaction rate is calculated with the following formula:

$$
R_{C}=\int_{0}^{\infty} \Phi(E) \cdot \sigma_{a c t} d E
$$

where $\Phi(E)$ is the neutron flux on the particular activation sample, $\sigma_{a c t}$ is the cross section of the corresponding neutron reaction. The activation reaction rate is then obtained by integrating over the spectral neutron distribution.

In the measurement the absolute activation reaction rate $\mathrm{R}$ is given by:

$$
\begin{gathered}
R=\frac{N\left(t_{0}\right)}{M \cdot b \cdot \Phi \cdot \varepsilon \cdot g} \\
N\left(t_{0}\right)=\frac{N_{12}}{e^{-\lambda\left(t_{1}-t_{0}\right)}\left[1-e^{-\lambda\left(t_{2}-t_{1}\right)}\right]} \\
\Phi=\frac{4 \pi}{\Delta \Omega} \cdot F_{\alpha} \cdot A_{\alpha}\left(\overline{E_{d}}, \phi_{\alpha}\right)
\end{gathered}
$$

where $N\left(t_{0}\right)$ is the $\gamma$-rate of a foil at $\mathrm{t}_{0} ; \mathrm{t}_{0}$ is the time when the irradiation is stopped, $\mathrm{t}_{1}$ is the time when the $\gamma$-rate measurement is started, $t_{2}$ is the time when the $\gamma$-rate measurement is stopped, $\lambda$ is the decay constant, $N_{12}$ is the total number of $\gamma$-counts from a foil in the measurement period $\left(t_{2}-t_{1}\right)$; $M$ is the total number of atoms in the foil, $b$ is the abundance of the isotope of interest; $\mathrm{g}$ is $\gamma$-ray emission ratio; $\varepsilon$ is the absolute efficiency of the high-purity germanium detector(HPGe), which is calibrated by means of a standard multi-line $\gamma$-ray source; $\Phi$ is the total neutron yield of the source. $A_{\alpha}\left(\bar{E}_{d}, \phi_{\alpha}\right)$ is the anisotropy correction coefficient as function of the average energy of the incident $\mathrm{D}^{+}$ion and the emission angle of the $\alpha$-particle; $\Delta \Omega$ is the solid angle covered by the detector to the titanium-tritium target. For $r$ (radius of the 
diaphragm in front of the detector) $<<\mathrm{R}$ (the distance from the center of the target to the diaphragm), the following relations are valid:

$$
\begin{gathered}
\Delta \Omega=\pi r^{2} / R^{2} \\
F_{\alpha}=\sum_{k=1}^{N} \rho_{\alpha k} \cdot e^{-(N-k) \cdot \lambda \cdot t_{\alpha}}
\end{gathered}
$$

where $F_{\alpha}$ are the weighted counts of $\alpha$-particles; $\rho_{\alpha k}$ are the $\alpha$-particle counts in the $\mathrm{k}^{\text {th }}$ time interval $t_{\alpha}$ and $e^{-(N-k) \cdot \lambda \cdot t_{\alpha}}$ is the decay coefficient. In this way, the absolute activation reaction rate is normalized to one source neutron and one atom of a foil.

Neutron reflectivities $\mathrm{K}$ characterise the reflected neutrons and are defined as a ratio:

$$
K=R_{y} / R_{n}
$$

Where $R_{y}, R_{n}$ are the absolute activation reaction rates with and without the $\mathrm{Al}$ reflector, respectively.

\section{Experimental system}

\subsection{Experimental set-up}

The experimental set-up is made of a D-T neutron source, shielding-moderator and a iron spherical shell. An Al shell is used as the reflector. The thickness of the iron shell is $10 \mathrm{~mm}$. The shielding-moderator as well as the neutron source and the reflector are arranged along a common axis. The set-up is illustrated in Figure 1.

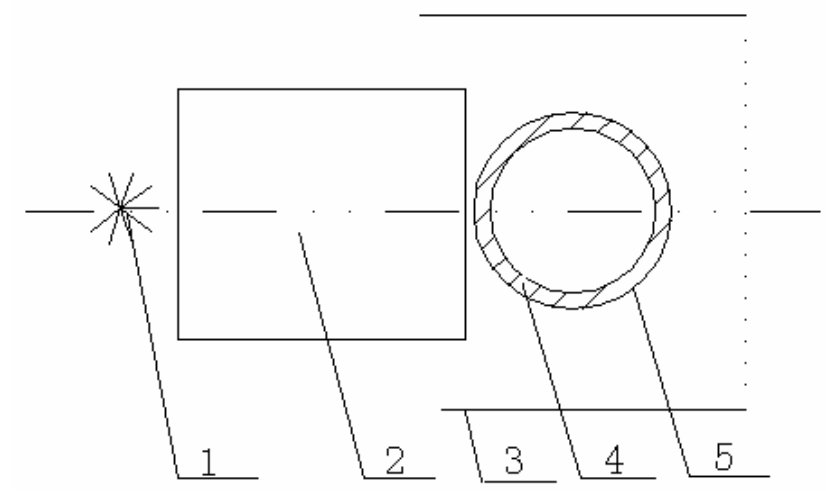

Fig.1 Experimental set-up

1-D-T neutron source, 2-shielding-moderator, 3-Al reflector, 4-iron spherical shell, 5-equatorial positions.

\subsection{Neutron source}

The primary $14 \mathrm{MeV}$ neutrons are produced by means of the D-T reaction with the K-400 neutron generator of our labratory. The beam energy of the $\mathrm{D}^{+}$ion is about $250 \mathrm{keV}$ and its average energy in the active volume of the target is about $134 \mathrm{keV}$. The area of the titanium- 
tritium on a copper backing is $12 \mathrm{~mm}$ in diameter. The target surface is water-cooled. The yield of source neutrons is obtained on absolute scale by monitoring the counts of the associated $\alpha$ particles measured with a semiconductor detector which is positioned at an angle of 135 degree to the beam direction. In order to account for a possible time-dependence of the neutron production, the $\alpha$-particle counters are automatically recorded in constant time intervals of $t_{\alpha}=30 \mathrm{~s}$ during the irradiation. The fraction of neutrons from the D-D reaction among the source neutrons is less than $1 \%$. The neutron yield amounts to $2 \sim 3 \times 10^{10} \mathrm{n} / \mathrm{s}$ when the $\mathrm{D}^{+}$ion beam current is $\sim 300 \mu \mathrm{A}$. The uncertainty of the absolute neutron yield measurement is less than $1.2 \%$.

\subsection{Detectors}

Six threshold detectors $\left(\mathrm{Cu}, \mathrm{Fe}, \mathrm{Al}, \mathrm{C}_{2} \mathrm{~F}_{4}, \mathrm{Zr}, \mathrm{Nb}, 0.2 \mathrm{~mm}, 0.3 \mathrm{~mm}, 0.6 \mathrm{~mm}, 1 \mathrm{~mm}, 0.2 \mathrm{~mm}\right.$, $0.2 \mathrm{~mm}$ in thickness, respectively) are used to measure the activation reaction rates. The nuclear characteristics of the activation foils are listed in Table 1 . The $\gamma$-rate of an irradiated foil is measured by means of an HPGe detector.

Table 1 Experimental parameters of the activation foils

\begin{tabular}{|c|c|c|c|c|c|c|}
\hline Reaction & $\begin{array}{c}\gamma \text {-ray } \\
\text { energy/keV }\end{array}$ & $\begin{array}{c}\text { Element } \\
\text { abundance }\end{array}$ & $\begin{array}{c}\gamma \text {-ray emission } \\
\text { rate }\end{array}$ & $\begin{array}{c}\text { Half- } \\
\text { life }\end{array}$ & $\begin{array}{c}\text { Threshold } \\
\text { energy/MeV }\end{array}$ & $\begin{array}{l}\text { Interfering } \\
\text { reaction }\end{array}$ \\
\hline${ }^{56} \mathrm{Fe}(\mathrm{n}, \mathrm{p}){ }^{56} \mathrm{Mn}$ & 846.6 & 0.9172 & 0.99 & $2.58 \mathrm{~h}$ & 5 & ${ }^{57} \mathrm{Fe}(\mathrm{n}, \mathrm{np}){ }^{56} \mathrm{Mn}$ \\
\hline${ }^{27} \mathrm{Al}(\mathrm{n}, \mathrm{a}){ }^{24} \mathrm{Na}$ & 1368.55 & 1 & 1 & $15.03 \mathrm{~h}$ & 6 & -- \\
\hline $\begin{array}{c}{ }^{93} \mathrm{Nb}(\mathrm{n}, 2 \mathrm{n}){ }^{92 \mathrm{~m}} \\
\mathrm{Nb}\end{array}$ & 934.53 & 1 & 0.955 & $10.16 \mathrm{~d}$ & 9 & -- \\
\hline${ }^{19} \mathrm{~F}(\mathrm{n}, 2 \mathrm{n}){ }^{18} \mathrm{~F}$ & 511 & 1 & 1.934 & $\begin{array}{l}109.8 \\
\min \end{array}$ & 11 & -- \\
\hline${ }^{90} \mathrm{Zr}(\mathrm{n}, 2 \mathrm{n}){ }^{89} \mathrm{Zr}$ & 909.2 & 0.5145 & 0.9987 & $78.44 \mathrm{~h}$ & 12 & -- \\
\hline${ }^{63} \mathrm{Cu}(\mathrm{n}, 2 \mathrm{n}){ }^{62} \mathrm{Cu}$ & 511 & 0.6917 & 1.96 & $\begin{array}{c}9.73 \mathrm{mi} \\
\mathrm{n}\end{array}$ & 12.6 & $\begin{array}{c}{ }^{63} \mathrm{Cu}(\mathrm{n}, \gamma){ }^{64} \mathrm{Cu} \\
{ }^{65} \mathrm{Cu}(\mathrm{n}, 2 \mathrm{n}){ }^{64} \mathrm{Cu}\end{array}$ \\
\hline
\end{tabular}

\section{Measurement and calculation}

\subsection{Experiments}

In the experiments the foils are placed between $0 \sim 180$ degree at a spacing of 30 degree on the surface of the iron shell at the horizontal equator. 0 degree is defined where the shell meets the moderator. To avoid perturbation between the detectors, every two detectors are mounted at an angle of 60 degree. Besides the reaction ${ }^{63} \mathrm{Cu}(\mathrm{n}, 2 \mathrm{n}){ }^{62} \mathrm{Cu}$ in natural copper there is also the reaction ${ }^{65} \mathrm{Cu}(\mathrm{n}, 2 \mathrm{n}){ }^{64} \mathrm{Cu}$ with a different decay time. This second reaction is discriminated by measuring the $\gamma$-rate of the $\mathrm{Cu}$ foils twice after an interval of 8 hours.

With a method combining experiments and $\mathrm{MC}$ calculation, the measurement technique of 
high-threshold detectors is systematically studied under special experimental conditions of small measurement space and small neutron flux. Several factors have to be considered for interpretation of the experimental data like self-absorption of the $\gamma$-rays and flux-attenuation of the neutrons in the foil, a perturbation among the detectors and scattered background neutrons.

The necessary corrections are calculated as function of a foil thickness. The thickness of each foil is optimized for minimal overall uncertainty. The influence of low-energy background neutrons is negligible for the activation detector used [4].

Measured reaction rates are shown in Figure 2. The evaluated total uncertainties of the measured rates are $5.2 \%$ to $6.1 \%$. They are due to the uncertainty of the source neutron yield $(1.2 \%)$, instability of instruments $(1.0 \%)$, detection efficiency of the HPGe detector $(4.0 \%)$, self-absorption of foils and flux-attenuation of neutrons (1.2\% 3.0\%), inaccurateness of the $\gamma$ ray peak's net area (1.5\%), statistical uncertainty of $\gamma$-ray counts $(0.8 \% \sim 1.9 \%)$, and some other minor contributions. The activation reaction rates have been normalized to one source neutron and one atom of a foil.



(a) with Al Reflector

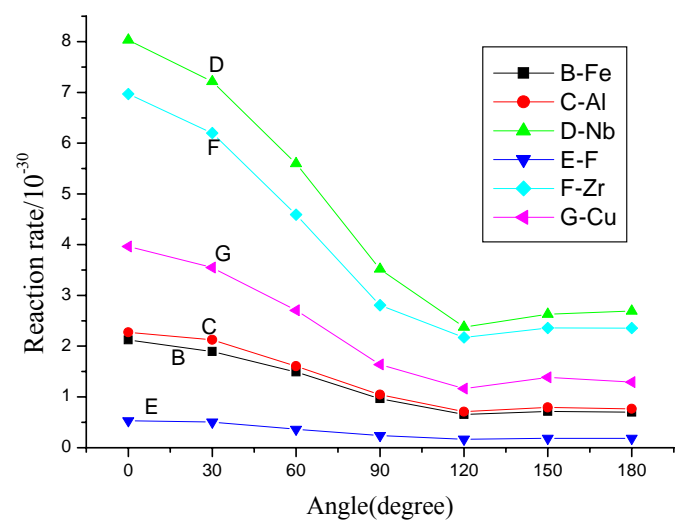

(b) without Al Reflector

Fig. 2 Measured absolute activation reaction rates

\subsection{Calculation with MCNP code}

To reduce the calculation time and improve the precision of the calculation, the sensitivity of the calculation on different nuclear databases, cut-off energies and tally types was investigated. When the effective reaction threshold is chosen as the cut-off energy, calculation time is considerably reduced. Comparing calculations with cell tallies (F4) with those performed with detector tallies (F5), the counting efficiency is higher and the results are slightly higher with cell tallies, but still credible. With different nuclear databases, calculated results can be slightly different.

The databases used in our calculations are listed in Table 2. Foil sizes and placement used in the calculation are the same as those in the experiments. The rates have also been normalized to one source neutron and one atom of a foil. The normalized reaction rates obtained by calculations with the MCNP/4B code and cell tallies F4 are shown in Fig. 3. 
Table 2 Databases in calculation with MCNP Code

\begin{tabular}{|c|c|c|c|c|c|c|}
\hline Activation foil & $\mathrm{Fe}$ & $\mathrm{Al}$ & $\mathrm{Nb}$ & $\mathrm{C}_{2} \mathrm{~F}_{4}$ & $\mathrm{Zr}$ & $\mathrm{Cu}$ \\
\hline Nuclear databse & Endf60 & Endf60 & Шdos & Endf60 & Шdos & Endf60 \\
\hline Source & B-VI.1 & B-VI.0 & LLNL/ACTL & B-VI.0 & LLNL/ACTL & B-VI.2 \\
\hline
\end{tabular}

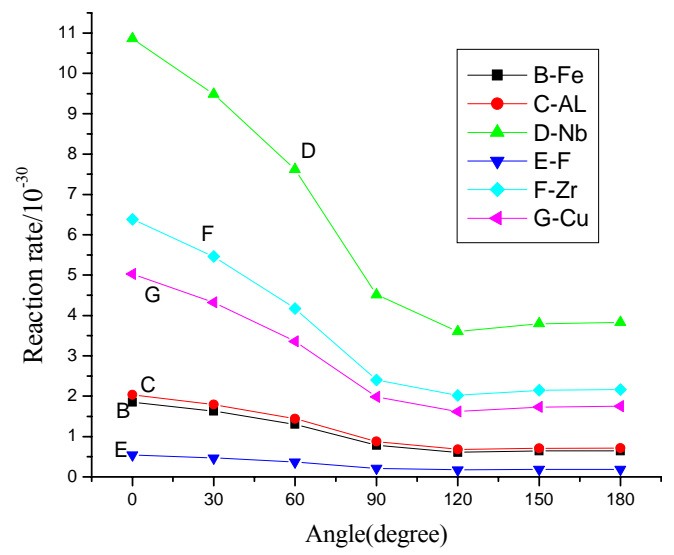

(a) with Al Reflector

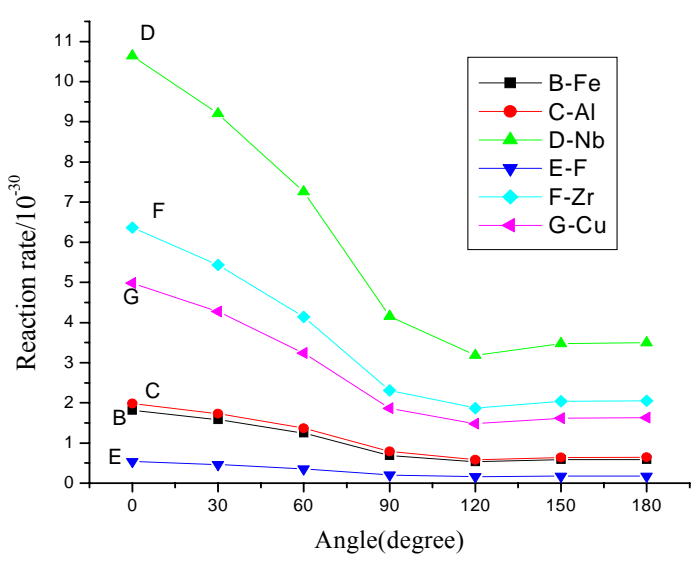

(b) without Al Reflector

Fig. 3 Calculated activation reaction rates with MCNP code

\section{Results and discussion}

With six high-threshold detectors $\mathrm{Fe}, \mathrm{Al}, \mathrm{Nb}, \mathrm{F}, \mathrm{Zr}, \mathrm{Cu}$, absolute activation reaction rates of high-energy neutrons are obtained with and without the Al reflector. For the different detectors the shape of the rates as function of the measurement angle is rather similar. Reaction rates are affected by the reaction threshold and the cross section. For a certain foil the rates at different positions are proportional to the neutron flux. The reproducibility of experimental results is within $4 \%$.

According to equ. (7), high-energy reflected neutron reflectivities are also obtained as the ratio of measured data and range from 1.0 to 1.14 . The total uncertainties of reflectivities are $3.4 \%$ to $4.2 \%$, fully correlated uncertainties like the uncertainty of the HPGe detection efficiency cancel out for the ratios. With increasing of angle the reflectivities increases for angles $<120 \mathrm{deg}$. and drops gradually for angles $>120 \mathrm{deg}$. With increasing threshold energy the values of the reflectivities decrease.

The calculated results of rates are compared with the experimental results and the discrepancies of rate values range from $12 \%$ to $30 \%$, except for $\mathrm{F}$ foils. It shows that some databases should to be re-evaluated. Simulated results of reflectivities range from 1 to 1.166. They are in accordance with the experimental results within the range of uncertainties at the majority of the measurement points.

Calculated reflectivities are shown in Fig. 4 as function of the threshold energy. 


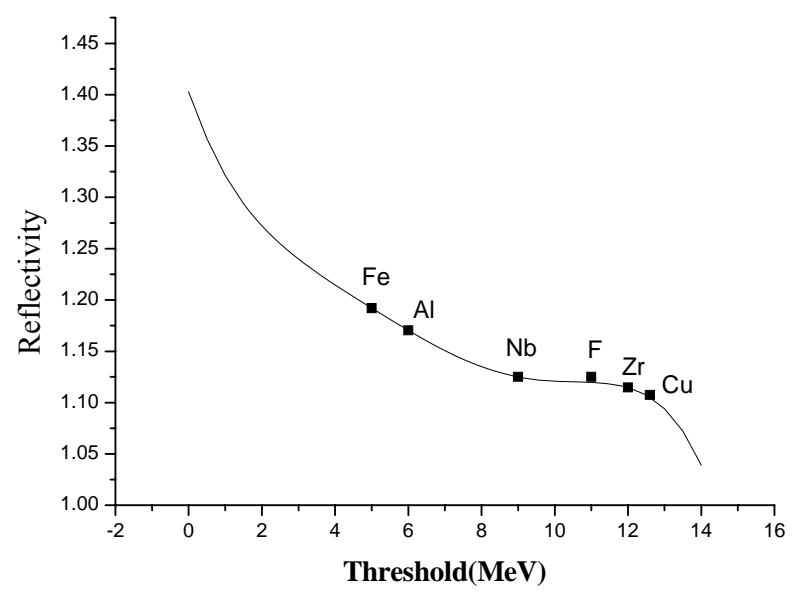

Fig.4 Calculated reflectivities as function of threshold energy

\section{Acknowledgements}

The authors would like to acknowledge the helpful discussions and assistance of Pro. Wang Dalun and Pro. Li Yijun.

\section{References}

[1] Walter Meyer, J.W.Leighty, J.W.Thiesing, Measured and Calculated Neutron Albedo for Concrete and Iron, Nuclear Science and Engineering 60 (1976) 405-420

[2] Monahan SP, The Neutron Physics of Concrete Reflectors: LA-UR-95-2196[R], USA: LA(1995)

[3] Liu Rong, Lin Libin, Wang Dalun, Li Yijun, Jiang Li, Wang Mei, Liu Chenglong, Yang Ke, Study on Technique for Measuring Reflection Neutrons by Uranium Fission Chamber, Nuclear Techniques, 23(2000) 851-855

[4] Lu Xinxin, Liu Rong, Jiang Li, Wang Mei, Lin Jufang, Wen Zhongwei, Wang Dalun, Study on the Measurement Technique of Fe Foil's Activated $\gamma$ Spectrum, Nuclear Electronics and Detection Technology, 25(2005) 104-107 